\title{
Non-Natural D-Amino Acids to Control Bacterial Virulence
}

\author{
Michael A. Bertucci ${ }^{1}$ and Kareem March ${ }^{2}$ \\ ${ }^{1}$ Department of Chemistry, Moravian College, Bethlehem, PA, 18018, USA; \\ ${ }^{2}$ Department of Chemistry, Hartwick College, Oneonta, NY, 13810, USA
}

\section{Introduction}

The progressive slowing of antibiotic development and the increase of bacterial drug resistances poses a major threat to human health [1]. Bacteria aggregate to form potent virulent communities bound by

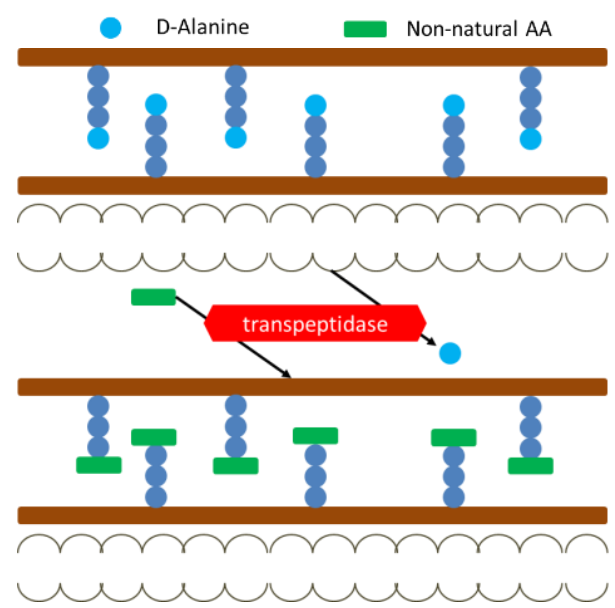

Fig. 1. The incorporation of non-natural amino acids into the peptidoglycan layer of the gram-positive bacterial cell wall.

an extracellular polymeric substance composed of sugars, proteins, and other secreted biomolecules. The resultant structure, a biofilm, is responsible for an estimated $80 \%$ of known bacterial infections. This formed barrier is resistant to changes in temperature, salinity, and antimicrobial agents, protecting proliferating cells on the interior of the structure. Thus, dismantling biofilms can serve as a means of impeding pathogenicity [2].

In 2010, Kolodkin-Gal and colleagues reported that D-amino acids are potent biofilm disruptors in gram-positive bacteria [3]. It was suggested that the terminal D-alanine residue of the gram-positive peptidoglycan layer can be replaced by another native or non-native amino acid via the promiscuous enzyme transpeptidase (Figure 1). The resultant substitution interferes with protein synthesis, leading to the eventual demise of the cell. This and other subsequent studies have divulged several amino acids capable of effective biofilm disassembly at low micromolar concentrations, with D-tyrosine as one of the most potent [4]. However, no clear correlation between amino acid structure and activity has emerged. Further, only the enantiomers of the twenty canonical amino acids have been screened, providing optimal space for the development of synthetically modified side chain residues to enhance potency.

In this study, we report a preliminary investigation into synthetic D-amino acids for biofilm disruption. Modified from a parent glutamic acid structure, three novel amino acids were prepared and screened in cultures of the opportunistic pathogen $S$. epidermidis to probe their antimicrobial capabilities.

\section{Results and Discussion}

To expediently develop a small set of novel D-amino acids for screening, glutamic acid was used as a handle for coupling a variety of primary and/or secondary amines to afford a de novo amide bond. Inspiration for the selection of amine coupling partners was taken from previously reported biofilm disruptors, like D-tyrosine and D-leucine that contain aromatic or larger aliphatic side chains (Figure 2) [4]. The amines were added to a cooled solution of 1-ethyl-3-(3-dimethylaminopropyl)carbodiimide (EDC), triethylamine, and Boc-D-glutamic acid methyl ester to yield the corresponding protected amino acid. In the presence of concentrated hydrochloric acid, the Boc and methyl ester moieties were cleaved to produce the free amino acid.

Solutions of the purified amino acids $(0.4 \mu \mathrm{M}$ to $400 \mu \mathrm{M})$ were prepared by serial dilution and combined with $S$. epidermidis biofilms, grown for $24 \mathrm{~h}$, in a simple crystal violet well-plate assay. After another $24 \mathrm{~h}$ incubation, the plates were read at $580 \mathrm{~nm}$ to determine the minimum inhibitory concentration of each synthetic amino acid. The combined data from 8 replicate trials reveals only modest variation in the biofilm surface (Figure 3). Both KM01 and the positive control D-tyrosine 
displayed a small but global reduction in absorbance, while KM02 and EM01 showed no significant decrease in biofilm density relative to the control. The discrepancy in the activity from $\mathrm{D}$ tyrosine and KM01 to KM02 implies that the carboxylate moiety in KM02 reduces biofilm disassembly, perhaps indicating the benefit of an electronrich arene.

However, a weak correlation was displayed between amino acid concentration and antibiofilm activity. In addition, the magnitude of biofilm reduction in all cases is minimal compared to the degree of biofilm disassembly described in previous reports. Amino acids, such as Dtyrosine, were shown to eradicate<smiles>[R]NC(=O)CC[C@H](NC(=O)OC(C)(C)C)C(=O)OC</smiles><smiles>[R]NC(=O)CC[C@H](NC(=O)OC(C)(C)C)C(=O)OC</smiles>

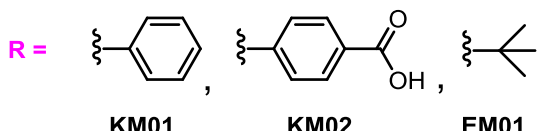

Fig. 2. Scheme for the synthesis of D-glutamic acid analogs KMO1, KMO2, and EMO1.

biofilms at concentrations as low as 10

$\mu \mathrm{M}$, which was simply not represented in our control experiments [4,5]. A study published concurrently by Sarkar and colleagues cites similar difficulties obtaining comparable potencies as those initially reported for D-amino acid mediated biofilm disassembly. After screening 96 natural and non-natural D-amino acids in three species of gram-positive pathogens (S. epidermidis, S. aureus, and $B$. subtilis), no significant biofilm reduction was observed [5]. These results call into question the effectiveness of employing D-amino acids as antimicrobials.

Despite the dissention in the field regarding the potential of D-amino acids to serve as biofilm disruptors, the pathway for the incorporation of non-natural amino acids into the peptidoglycan layer of gram-positive bacteria has been verified. The well-known epitope 2,4-dinitrophenylalanine (DNP) was introduced into the internal oligopeptides of $B$. subtilis, promoting assembly of endogenous antibodies at the cell surface [6]. The flexibility of the transpeptidase enzyme mediating this process

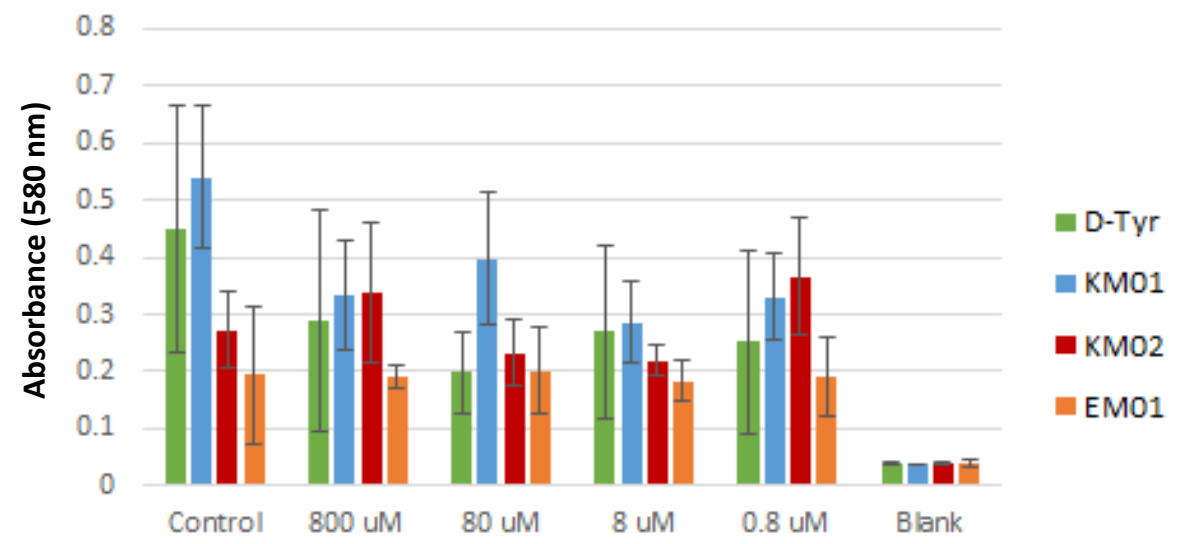

Amino Acid Concentration

Fig. 3. Decrease in S. epidermidis biofilm density (absorbance) after incubated for $24 \mathrm{~h}$ with D-tyrosine and three D-glutamic acid derivatives KMO1, KMO2, and EM01. 
was further employed to modify extracellular peptides at their $C$-termini, largely altering the topography of the bacterial cell wall [7].

In this initial investigation of synthetic $\mathrm{D}$-amino acids as biofilm disruptors, three novel glutamic acid derivatives were designed and screened in S. epidermidis. Although the analogs only displayed slight perturbations in biofilm densities, the feasibility of inserting synthetically modified amino acids into the gram-positive cell wall has been verified in the literature. By integrating previous research in peptide science and chemical biology in bacteria, the peptidoglycan layer remains an open canvas for antimicrobial decoration.

\section{Acknowledgments}

We would like to thank the Department of Chemistry at Hartwick College and the Rochester Academy of Sciences for funding as well as Professor Mary Allen for the materials and facility to conduct the biofilm degradation assays. Future work on the project will be complete with support from the Department of Chemistry at Moravian College.

\section{References}

1. Centers for Disease Control and Prevention. (2013) Drug Resistance. Retrieved from http://www.cdc.gov/media/releases/2013/p0916-untreatable.html

2. Costerton, J.W., Stewart, P.S., Greenberg, E.P. Science 284, 1318-1322 (1999), http://dx.doi.org/10.1126/science.284.5418.1318

3. Kolodkin-Gal, I., et al. Science 328, 627-629 (2010), http://dx.doi.org/10.1126/science.1188628

4. Leiman, S.A., et al. J. Bacteriology 195, 5391-5395 (2013), http://dx.doi.org/10.1128/jb.00975-13

5. Sarkar, S., Pires, M.M. PLOS One 10 (2015), http://dx.doi.org/10.1371/journal.pone.0117613

6. Fura, J.M., Sabulski M.J., Pires, M.M. ACS Chem. Biol. 9, 1480-1489 (2014), http://dx.doi.org/10.1021/cb5002685

7. Pidgeon, S.E., et al. ACIE 54, 6158-6162 (2015), http://dx.doi.org/10.1002/anie.201409927 